\title{
Varying Pause Time Effect on AODV, DSR and DSDV Performance
}

\author{
${ }^{1}$ Zafar Mahmood, ${ }^{1}$ Muhammad Awais Nawaz, ${ }^{2}$ Dr Mudassar Iqbal, ${ }^{3}$ Saleem Khan, ${ }^{4}$ Zia ul \\ Haq \\ ${ }^{1,2,4}$ Faculty of Computer Science and information Technology, University of Gujrat, ${ }^{3}$ Comstat Institute \\ of Information Technology
}

\begin{abstract}
A mobile ad hoc network is a collection of wireless nodes without any fixed structure and connected with each other through wireless links. MANETs have self-organized topology in which mobile nodes are free to move, as a result establishing a stable and reliable network is a difficult and critical task. Nodes in Nobile Ad-hoc network acts as sender, receiver and router for communicating data and control packets. Using NS-2 simulator Performance of AODV, DSDV and DSR is analyzed under varying pause time in this paper. Performance of these routing protocols are analyzed on the base of following performance metrics: packet delivery ratio, average end to end delay, throughput, no of sent packets, no of receive packets, no of routing packets, normalized routing load and no of dropped packets under variant Pause time. Results of Comparison analysis of Routing Protocols are generated with the help of Simulation in the form of graphs. The simulation result shows the significance of each protocol in different situations. We believe that this study will provide comprehensive comparative study of AODV, DSR and DSDV based on varying pause time at one place, which will help the researcher to further investigate any particular metrics value of particular protocol.
\end{abstract}

Index Terms: Mobile Ad hoc Networks (MANETs), Ad Hoc on Demand Routing Vector (AODV), Dynamic Source Routing (DSR), Destination Sequenced Distance Vector (DSDV), network simulator NS-2.

(C) 2015 Published by MECS Publisher. Selection and/or peer review under responsibility of the Research Association of Modern Education and Computer Science

\section{Introduction}

Mobile ad hoc network is a collection of wireless nodes without any fixed structure and connected with each other through wireless links. For past many years different researchers studied major routing protocols, AODV [1], DSDV [2] and DSR [3] and their working mechanism. MANETs are self-configured networks mostly established in the situation where installation of infrastructure based network is expensive or difficult to implement like in battle fields, rescue operations and undersea operations [4]. A wireless Ad-hoc network is built by connecting a set of mobile devices/mobile user or devices through wireless links that is spread over a

* Corresponding author. Tel.:

E-mail address:Zafar.mehmood@uog.edu.pk;awais00772@hotmail.com;m.iqbal@uog.edu.pk;zia@uog.edu.pk;

skhan.ciit@gmail.com 
certain geographical area.

A wireless mobile Ad-hoc network don't need a fixed and predefined infrastructure and topology to keep the network connected, due to the frequently changing network structure nodes in the network supposed to be leave, join or merged together to form a separate network. The physical structure (topology) in such a network keeps varying frequently. Since all the nodes in the network is mobile, and without centralized administration mechanism, the network structure is created by connecting all nodes with each other on behalf of their own responsibility. Due to the mobility of nodes a node may leave or join the network by moving freely in the network area by establishing connection with other node, providing the router behavior for network service [5]. As a result of mobility of nodes/devices and changing topology mobile Ad-hoc network may be very diverse from each other with respect to topology and area of application. Since no centralized command and control system are required for mobile Ad-hoc network, installation of Ad-hoc networks can be easy and fastly, without the implementation of any advance development or construction of expensive network substructure. Due to these reasons, Ad-hoc network is a first choice for particularly those applications where such setup and structure is either inaccessible or untrustworthy. The IETF ("Internet engineering Task Force") as Mobile Adhoc networking, working group works precisely to develop different Routing Protocols topologies based on IP, to improve and enhance mobile routing and interface definition for use within the internet protocol suit [6].

Many researchers have analyzed the performance of AODV, DSDV and DSR by using different performance metrics under different circumstances. To analyze the performance of AODV, DSDV and DSR using their different metrics values under varying pause time is the main objective of this research.

Under predefined scenario and constraints like simulation area 500*500, 100 mobile nodes, maximum connections 20,512 bytes packets size, having fixed mobility of $20 \mathrm{~m} / \mathrm{s}$ with varying pause time from 10 to 50 seconds. Different performance metrics like throughput, average end to end delay, packet delivery ratio, no of sent packets, no of receive packets, no of routing packets, normalized routing load and no of dropped packets

are compared for these protocols.

In this paper we have compared 9 performance metrics value. In past research few parameters are measured for ad hoc routing protocols. Through the conclusion of this research future researchers can find a complete analysis of these routing protocols in a single research paper.

The remaining of this paper is structured as follows. Section 2 covers brief description about related work. In section 3 different routing protocols are discussed. Methodology, simulation tools, simulation setup is described in detail in section 4. Section 5 shows the result of our research and its analysis. Finally in section 6 the conclusion of our research is given.

\section{Add-hoc Routing Protocols}

Mobile ad-hoc networks routing protocols are classified in two core types: On demand routing protocols and table driven routing protocols. Dynamic source routing protocol (DSR) and Ad hoc on demand Distance Vector (AODV) are the examples of Reactive protocols and Destination Sequenced Distance Vector (DSDV) is the example of Proactive Routing Protocol. Every routing protocol has its advantage and disadvantages. Every one of these protocol shows best performance in different scenario. A single protocol is not best in every environment.

\subsection{Ad hoc on-demand Distance Vector (AODV)}

AODV is one of the most prominent reactive protocols. It is on demand protocol but don't use the concept of source routing rather it is based on hop by bop count. Every node maintains the routing information by using routing table which is maintained at every node of the network. In routing table, destination address, next hop IP address and destination sequence number is stored. Route request (RREQ), Hello message, Route reply (RREP) and Route Error (RERR) are the four types of messages used in AODV mechanism. Hello message is used to monitor links, every node in network broadcast the Hello Message, and this hello message will be 
receive by all neighbour nodes. Invalid Links are detected if node fails to receive any of the control or data packet message. Route Request is used when a source $\mathrm{S}$ wants to send data to Destination $\mathrm{D}$, this request is broadcasted to neighbours. All the intermediate nodes between source and destination broadcast the request in two cases, one they don't receive the request before or second it is not the destination node. If intermediate is the destination or it have the route to destination then it will reply to source in hop-to-hop fashion. Each intermediate node create a route to destination with Rote request receives. Data sending starts when source receives the destination route [7] [8]. Since source and all intermediate nodes uses broadcast mechanism there may a chance of receiving multiple copy of route request or route reply, in this case hop by hop count process will be used. Least hop count route is selected for data transfer. If a link remains idle for a certain period, link failure chance may happen Source invalidates the route after receiving the route error. Process for route discovery again initiates if requires.

\subsection{Dynamic source routing (DSR)}

DSR is another well-known reactive routing protocol originally developed by the Johnson, Maltz and Broach. Source routing is key feature of DSR [9]. DSR protocol is source based routing protocol. Using hops or intermediate nodes data can be transfer from source node to destination node. Two main concepts are used by DSR, route discovery and route maintenance, in route discovery process the source and destination node find and establish a route through RREQ and RREQ process. Suppose a node S want to communicate with a destination node $\mathrm{D}$, first the source node will check its cache for any available valid route, if a valid path exist in source node cache it start communicating with the destination node, and if it didn't find any path in its cache, the source node start a process for route discovery to find a path to the target destination node by generating a ROUTE REQUEST packet, having several information like destination id number, sequence number, source node id and request id. Intermediate node after receiving the ROUTE REQUEST performs one of the following actions, if it is a destination node by comparing the sequence number generates ROUTE REPLY; the intermediate node decides whether it has recently seen this request? If yes simply discard the REQUEST, in third action the intermediate node append its information to the packet header and rebroadcast the ROUTE REQUEST. Following the above process when the route request reaches at destination route reply process is started back to the source node. All the information related to sourced node, intermediate and destination node are stored in route reply packet, which is cached by source node for sending data packets and further communication

The method through which a node checks either the route is still valid or not is called route maintenance process. In case of link error an upstream node generate a route error message to inform the source node having problems in forwarding data or control message. But before informing the source node the node identifying link error checks its route cache, as there any other path available to retransmit the data or control packets if available use this route if not notify to source node. The source node then checks its cache for a new route, if available use it, if not again flood a route request message to all the nodes. If route maintenance that source route is broken then node $\mathrm{S}$ will again flood the ROUTE REQUEST (RREQ). Using initiation id and route request id, route request us uniquely identified. To minimize ROUTE REQUEST messages every node that received ROUTE REQUEST first check either route record is present in it ROUTE CACHE or not. Reply to ROUTE REQUEST is sent by destination node if it has already information of destination or it is the destination. Route Error and Ack's are used in the process of route maintenance. Source node receives a Route error message if error encounters. After receiving the route error message route discovery process again starts [10].

\subsection{Destination Sequenced Distance Vector (DSDV)}

DSDV is table driven routing protocol using Bellman Ford algorithm to calculate a path. As DSDV uses proactive approach all nodes in the network knows a route to every other node in the network prior to 
communication. For this purpose every node in the network broadcast a HELLO packet at regular interval to get information about the neighbour nodes, route available to reach the neighbour node and to sense the valid or invalid link. A routing table is maintained at every node to store all these information. Every destination route is associated with a label of sequence number. Considering a route for sending data is based on sequence number, a route having higher sequence number associated will be preferred for communication. Using routing table data and control information is transmitted throughout the network to transmit the updated information about the network topology and node status [11] [12] [13]. As DSDV is a proactive protocol, routing table need to be updated, changes in the network can also be managed by exchanging routing table between the all nodes. Even and odd sequence number method is used to check that either link is alive or broken. Even sequence number is used to represent link is alive; if sequence number is odd link is broken. In DSDV finding the least hop count route to destination from source is the major goal. Battery consumption is the major problem of proactive protocols because they always require battery in order to update routing table [12] [13].

\section{Related Work}

In paper [14], the author describe a comprehensive comparison of different ad hoc routing protocols DSDV, DSR, AODV and TORA under varying pause time and fixed number of nodes, has been carried using NS-2 as a network simulator. Different performance metrics like packet delivery ratio, number of hops taken by packet to reach destination and routing overhead are compared for different protocols. Pause time varies from 0 to 900 sec: $0,30,60,120,300,600$ and 900 are the seven different value for simulation, with 50 numbers of nodes during the simulation. 70 different patterns of movements are used, 10 for each value of pause time. Two different values for movement was also declared $1 \mathrm{~m} / \mathrm{s}$ and $20 \mathrm{~m} / \mathrm{s}$. result shows that when mobility was $1 \mathrm{~m} / \mathrm{s}$ and pause time was $900 \mathrm{sec}$ all protocols delivers maximum number of data packets. When pause time value decreases to 300 or less. Overhead value increases. DSR have the minimum overhead TORA has the maximum. DSDV and DSR shows best performance when mobility is low and pause time is high while TORA shows the worst performance. AODV performance is better than DSDV and TORA but its performance is gradually decreases with the increasing mobility.

In paper [15] comparison for AODV, DSDV and DSR are carried using different mobility models. For mobile nodes movement NS-2 nodes movement generator is used. Following pause time values are used for the mobility variation $0,50,100,150,300,600$ and 900. Normalized routing load and packet delivery fraction are the two metrics values for which comparison has been made. 20, 50 and 70 number of nodes is used in three different scenarios with the variation of pause time. Result shows that for packet delivery fraction with less dense environment AODV outclass DSR and DSDV. At node 50 AODV and DSDV shows almost the same performance. When number of nodes are 70 all protocol unable to deliver more than $70 \%$ data packets. In case of normalized routing load DSDV shows minimum routing load in all cases; when number of nodes are 20, 50 or 70. With less number of sources DSR and AODV shows almost same routing load, but with the increases of sources this value decreases for DSR, because of more route requests AODV has maximum value of routing load than DSR and DSDV. The author made a conclusion that with less mobility and small number of resources DSDV shows better performance than DSR and AODV.

In paper [16] author compares the performance of DSR and AODV on the base of three performance metrics: packet delivery ratio normalized routing load and end to end delay. NS-2 is used for the variation of network load, network variation and network size. Result shows that performance of DSR is better than AODV. At the rate of 4 packets per second by using 10, 20,30 and 40 number of sources is used, at 10 and 20 sources PDF value is almost same for both AODV and DSR. But with the increase of resources and mobility DSR starts losing data packets. Result of this simulation shows that in less stressful situation DSR outclass the AODV. DSR have less routing load but AODV have better performance is more stressful situation. Aggressive caching and fail to fresh the route cause delay issues in DSR but at the same it helps the DSR in low load environment.

In paper [17] throughput, delay normalized routing load, number of sent packets, of three routing protocols AODV, DSDV and DSR are analysed under fixed mobility and variant number of nodes using NS-2 Simulator. 
The parameters according to which simulation is carried out are $500 * 500$ terrain area, mobility is fixed $20 \mathrm{~m} / \mathrm{s}$, number of nodes varies from 20 to 40 and simulation time is $200 \mathrm{sec}$. The result shows that DSR have better performance than AODV and DSDV in less dense environment but when the number of nodes increases the DSR performance gradually decreases.

In reference [18], table base routing protocol AODV and source base routing protocol DSR are compared under predefined constraints. Terrain area changes during simulation from $100 * 100$ to $1000 * 1000$ and pause time values are $0,10,20,30,40,50$ second, other simulation parameters which are used in simulation are number of nodes 100 , mobility $10 \mathrm{~m} / \mathrm{s}$, bandwidth $2 \mathrm{mbps}$, simulation time $50 \mathrm{~second}$, and transmission range is 250 meter. Ns-2 is used as a network simulator for the simulation. Different performance metrics like number of sent packets, number of received packets and ratio of received to send packets are to be measured in this research. Result shows that in terrain area $100 * 100$ DSR shows most favourable behaviour than $1000 * 1000$. AODV also shows best performance in small terrain area i.e. 100*100. While comparing the AODV and DSR, performance of DSR in $100 * 100$ is better than its $1000 * 1000$ and also better than the AODV performance in $100 * 100$ and $1000 * 1000$ terrain area.

\section{Methodology}

In this portion we describe the network simulation tool, metric values, parameters which we have used for the performance analysis of AODV, DSDV and DSR with the variation of number of nodes.

\subsection{Simulation Tool}

Simulation is basically the designing and making a model of physical system, experimenting, evaluating and analyzing this system in different environments to check its behavior. Different types of simulation tools are used by the researchers for simulation according to their requirement of research. In this research paper we use network simulator NS-2 which is a discrete simulator and object oriented based on both $\mathrm{C}++$ and OTcl. Ns-2 also performs the simulation on the base of two type of file C++ and TCL. It is used for both wired and wireless protocols and also support user extended protocols with IP protocol. Ns-2 has many other suitable features like a channel for wireless, routing along multiple paths etc. [19] [20].

\subsection{Metrics Value}

To evaluate the performance of both proactive and reactive protocols different metrics values like: Throughput, end to end delay, packet delivery ratio, normalized routing load, number of dropped packets, number of dropped (bytes), number of sent packets, number of received packets has been evaluated.

\subsection{Simulation Setup}

In this paper we have analysed the number of sent packets ,number of received packets, number of routing packets, packet delivery ratio, normalized routing load, number of dropped data packets, number of dropped data bytes, average end to end delay and average throughput using the comparison of AODV, DSDV and DSR routing protocols where the total number of nodes that are used are 100 with maximum 20 connections, pause time varies from 10 to $50 \mathrm{sec}$, traffic type CBR, maximum packet size used in simulation is 512 Bytes, and the mobility $20 \mathrm{~m} / \mathrm{s}$. The scenario size which we have used is $500 * 500 \mathrm{~m}$. two ray ground propagation is used in wireless channel with Omni antenna and 802-11 Mac Type. The simulation is taken to be of $200 \mathrm{sec}$ [21] [22] [23]. 
Table 1 Performance Parameter Simulation Parameters Values

\begin{tabular}{|l|l|}
\hline Number of Nodes & 100 \\
\hline \hline Pause Time & $10,20,30,40,50 \mathrm{sec}$ \\
\hline \hline Simulation Time & $200 \mathrm{sec}$ \\
\hline \hline Mobility & $20 \mathrm{~m} / \mathrm{s}$ \\
\hline \hline Traffic Type & CBR \\
\hline \hline Packet Size & 512 Bytes \\
\hline \hline Scenario Size & $500 * 500 \mathrm{~m}$ \\
\hline \hline Channel Type & Wireless Channel \\
\hline \hline Radio Propagation Model & Two Ray Ground \\
\hline \hline Mac Type & $802-11$ \\
\hline \hline Link Layer Type & LL \\
\hline \hline Maximum Packets & 50 \\
\hline \hline Protocols & AODV,DSDV,DSR \\
\hline \hline Number of Connections & 20 \\
\hline \hline Antenna model & Omni Antenna \\
\hline \hline
\end{tabular}

\section{Result and its Analysis}

In this section results after comparison for all the three protocols AODV, DSR and DSDV have been plotted using graphs, following the explanation.

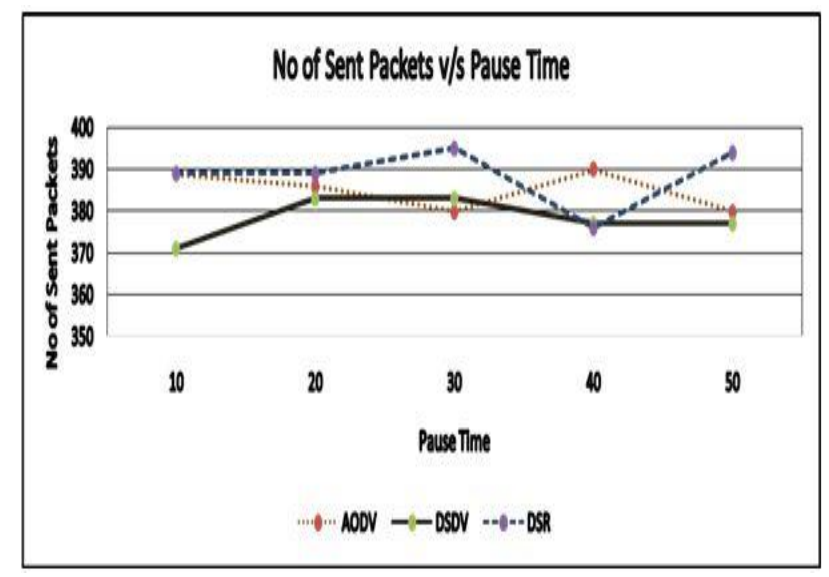

Fig. 1. No of Sent Packets v/s Pause Time

Under the following constraints of performance parameter, when numbers of nodes are 100 , mobility $20 \mathrm{~m} / \mathrm{s}$, pause time varies from 10 seconds to 50 seconds, packet size 512 byte, time for which simulation is done is 200 seconds, the fig. 2 shows that all protocols have variation in packets sending. DSDV shows poor performance for packets sending rate under limited pause time as compared to DSR and AODV, because in starting, it have to update the routes and all nodes but its performance gradually improves with the increasing pause time as all information about the route and intermediated nodes are available. Overall DSR shows better performance than DSDV and AODV. When the pause time is $10 \mathrm{~m} / \mathrm{s}$ then network structure changes rapidly, since DSR and AODV have a mechanism to adopt the network change quickly and with the increase of pause time there is 
variation in their performance. With the increasing pause time network topological structure getting decreases as a result DSDV performance getting improved by using its previously stored routing table information.

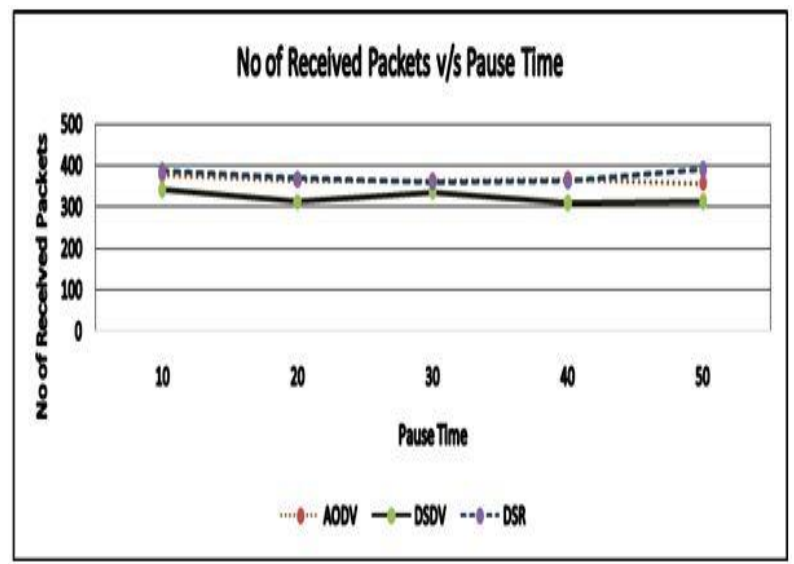

Fig. 2. Number of Received Packets v/s Pause Time

Under the following constraints of performance parameter, when numbers of nodes are 100, mobility $20 \mathrm{~m} / \mathrm{s}$, pause time varies from 10 seconds to 50 seconds, packet size 512 byte, time for which simulation is done is 200 seconds, the fig. 3 shows that all protocols have almost the same result, and have less variation with the changing pause time value. With the increase of pause time network become more and more stable and network changes occurs slowly, as a result graph demonstrates a stable behaviour for the three protocols. At the start of simulation under limited pause time since network structure changes frequently DSDV shows poor performance as compared to DSR and AODV, as every time when the network structure changes every node in the network must be updated. By comparing the results for DSR and AODV, DSR shows slightly better outcome because of its aggressive cache using while AODV use only one route from source to destination.

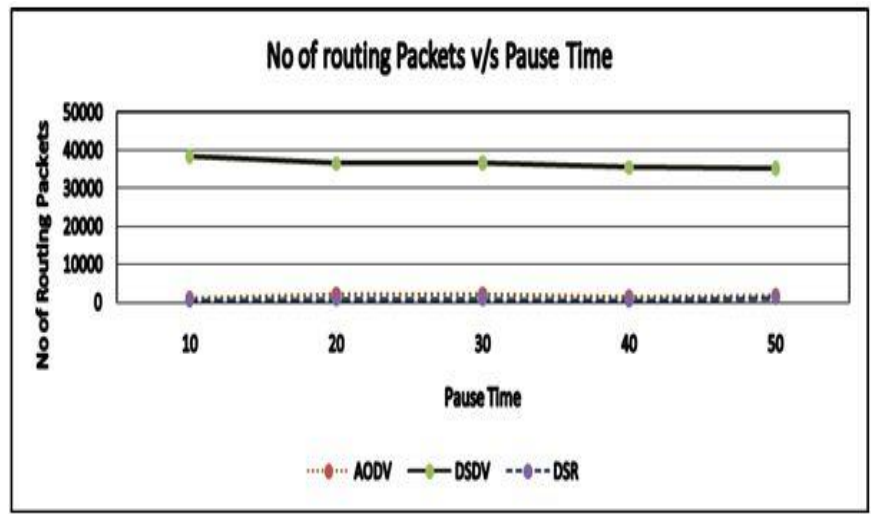

Fig. 3. Number of Routing Packets v/s Pause Time

Under the following constraints of performance parameter, when numbers of nodes are 100, mobility $20 \mathrm{~m} / \mathrm{s}$, pause time varies from 10 seconds to 50 seconds, packet size 512 byte, time for which simulation is done is 200 seconds, fig. 4 shows a huge difference between table driven routing protocol DSDV and on demand routing protocols, DSR and AODV. Since all table driven routing protocols needs to propagates a HELLO message 
after regular interval regardless communication is taking place or not, due to which it generate a huge number of routing packets. The reason for such difference in values of AODV, DSR from DSDV is the proactive approach of DSDV. Large numbers of control messages are sent in DSDV in order to update the nodes. Proactively links are checked by all nodes. Continuous topology changing information due to varying pause time or mobility of nodes is also provided to all nodes. As AODV and DSR both are on demand routing protocols, needs to generates data and control packets when two nodes want to communicate with each other.

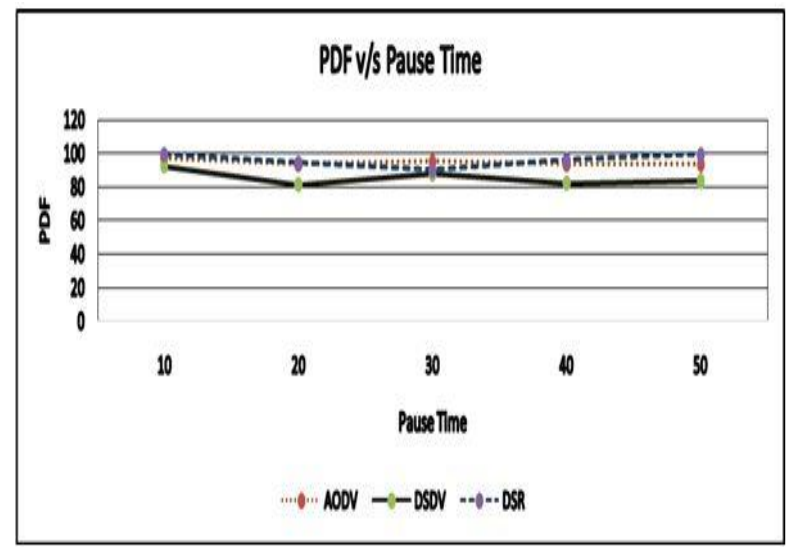

Fig. 4. Packet Delivery Fraction v/s Pause Time

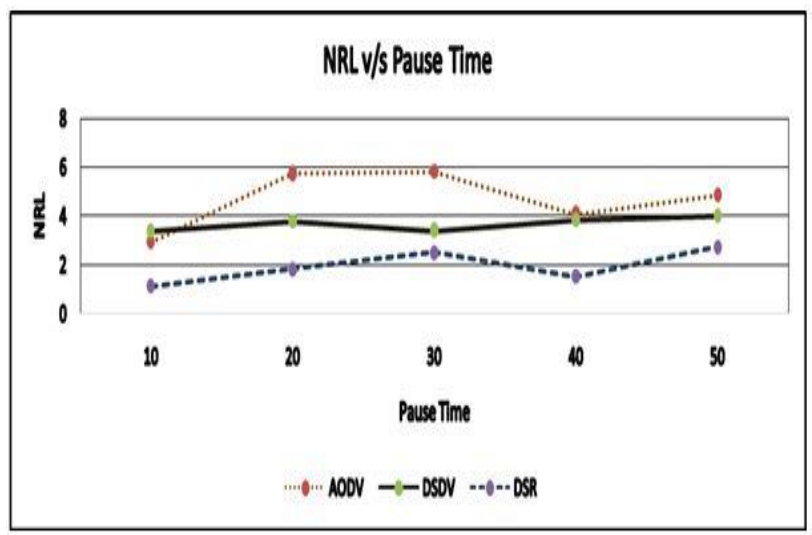

Fig. 5. Normalized Routing Load v/s Pause Time

Under the following constraints of performance parameter, when numbers of nodes are 100, mobility $20 \mathrm{~m} / \mathrm{s}$, pause time varies from 10 seconds to 50 seconds, packet size 512 byte, time for which simulation is done is 200 seconds, the fig. 5 shows almost stable and constant behaviour for AODV and DSR for packet delivery friction. As compared to DSDV which shows poor performance because of routing table mechanism which needs to be updated every time if changes occurs in network structure or a node leave or join the network. Single change in route must be forwarded to all the nodes due to which performance decreases. Time to live for packets expires if a valid and cost effective route is not selected well in time. Both DSR and AODV since using on demand nature, causing minimum number of packets to be dropped.

Under the following constraints of performance parameter, when numbers of nodes are 100 , mobility $20 \mathrm{~m} / \mathrm{s}$, pause time varies from 10 seconds to 50 seconds, packet size 512 byte and time for which simulation is done is 
200 seconds, fig. 6 shows that DSR outclass both AODV and DSDV for normalized routing load. Under higher mobility(limited pause time value) all the three protocols give good results for NRL, but with the increasing pause time NRL value is also getting increases. Reasons for DSR good performance is source base routing in which a source node include all the information about route, intermediate node and destination node. While in AODV since route knowledge is restricted only to the source of any routing packets being sent causing higher routing overhead. Thus AODV have to depend on route discovery flood more often, which may carry major network upstairs. While on the other hand, ROUTE REPLY establishes a large section of DSR's routing overhead. DSDV show poor performance because it is not adaptive as AODV and DSR in maintaining routing information.

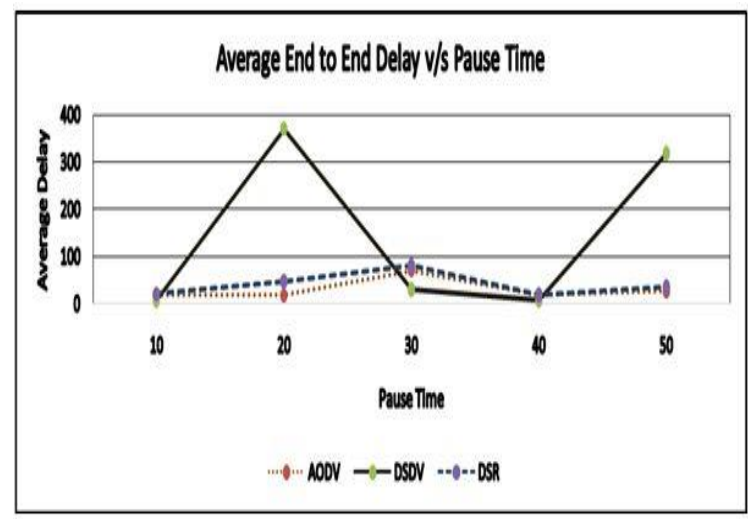

Fig. 6. Average End to End Delay v/s Pause Time

Under the following constraints of performance parameter, when numbers of nodes are 100, mobility $20 \mathrm{~m} / \mathrm{s}$, pause time varies from 10 seconds to 50 seconds, packet size 512 byte and time for which simulation is done is 200 seconds, the fig. 7 shows the average end to end delay. DSDV shows great variation in the result. At the start of simulation all the three routing protocols shows almost same end to end delay. Overall, high mobility causes increases the delay; but due to congestion delay increases in lower mobility. The delay time is also exaggerated by route discovery, In DSDV route building may not occur rapidly. This lead to extensive delays waiting or new routes to be resolute. In DSR route discovery is fast, therefore shows a better delay performance. DSR has relatively high delay than AODV, but it declines with increase in pause time.

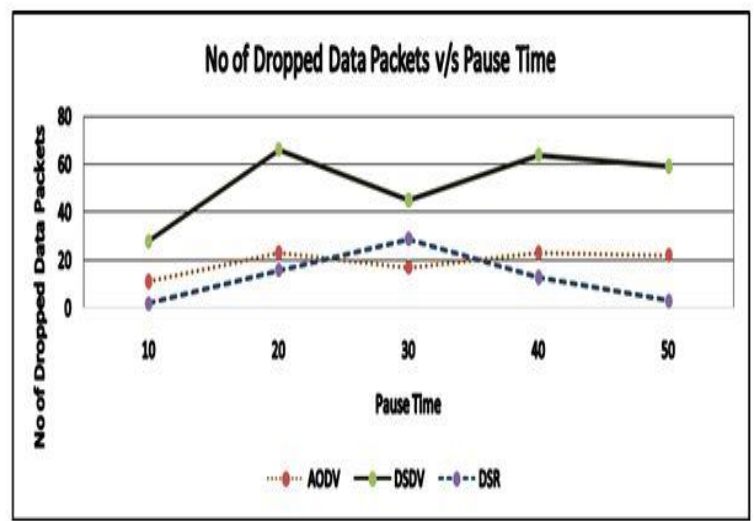

Fig. 7. Number of Dropped Packets v/s Pause Time 
Under the following constraints of performance parameter, when numbers of nodes are 100 , mobility $20 \mathrm{~m} / \mathrm{s}$, pause time varies from 10 seconds to 50 seconds, packet size 512 byte and time for which simulation is done is 200 seconds, the fig. 8 shows the simulation result of the routing protocols for the number of dropped data packets. In this graph all protocols shows variation in the performance. DSDV drops maximum number of data packets as compared to AODV and DSR. Performance of AODV getting increases till $20 \mathrm{~m} / \mathrm{s}$ pause time, then decreases for $30 \mathrm{~m} / \mathrm{s}$ and then again little bit increases. The main reason for the packets drops is time to live entry in packets header. If protocols take too much time to select the routes then packets will drop automatically because of short TTL. Packets dropping rate decreases, if protocol selects the route efficiently. Since DSR don't need any periodic update, as a result dropping packets rate is minimum as compared to AODV and DSDV.

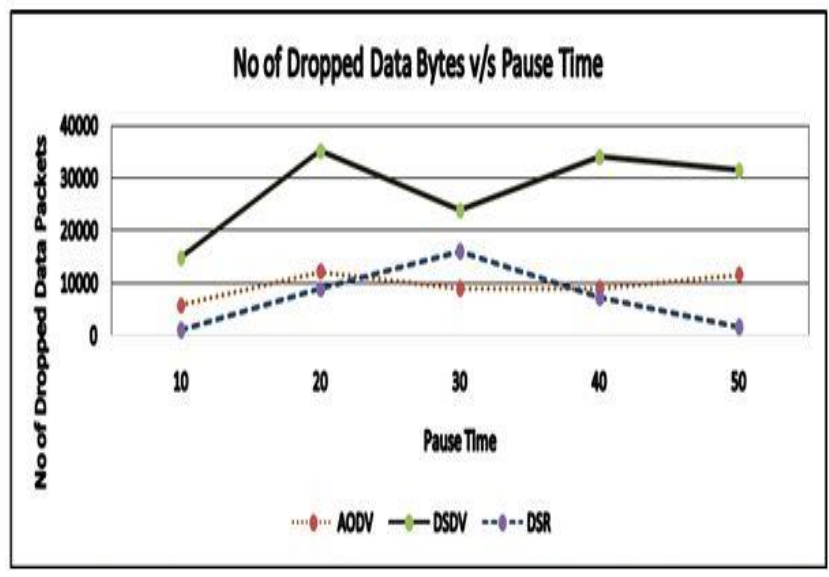

Fig. 8. Number of Dropped Bytes v/s Pause time

Under the following constraints of performance parameter, when numbers of nodes are 100 , mobility $20 \mathrm{~m} / \mathrm{s}$, pause time varies from 10 seconds to 50 seconds, packet size 512 byte and time for which simulation is done is 200 seconds, the fig. 9 shows almost same behaviour as described in figure 8 because number of dropped bytes is directly proportional to number of dropped packet.

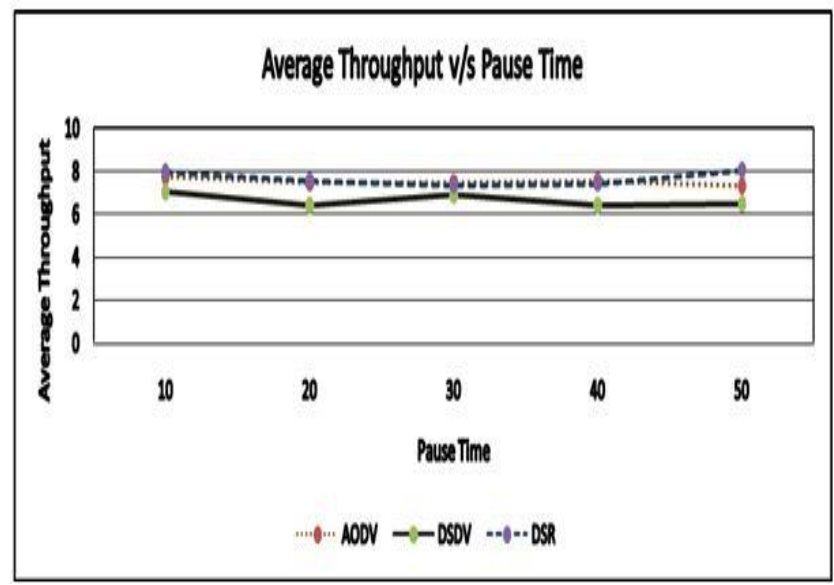

Fig. 9. Average Throughput v/s Pause Time 
Under the following constraints of performance parameter, when numbers of nodes are 100, mobility $20 \mathrm{~m} / \mathrm{s}$, pause time varies from 10 seconds to 50 seconds, packet size 512 byte and time for which simulation is done is 200 seconds, the fig. 10 shows almost stable and constant behaviour for AODV and DSR for average throughput. As compared to DSDV which shows poor performance because of routing table mechanism which needs to be updated every time if changes occurs in network structure or a node leave or join the network. Single change in route must be forwarded to all the nodes due to which performance decreases. Time to live for packets expires if a valid and cost effective route is not selected well in time. Both DSR and AODV since using on demand nature, causing minimum number of packets to be dropped, hence throughput will be increases.

\section{Conclusion}

The evaluation of existing routing approaches is necessary for analysing their performance. In this paper we carried out a comprehensive comparison by using NS-2 as a network simulator tool to analyses the behaviour of AODV, DSR and DSDV routing protocols by plotting the results in graphs. Multiple performance metrics are analysed like: number of sent packet, number of received packet, packet delivery ratio, and normalized routing load, no of dropped data packets, no of dropped data bytes, average end to end delay and throughput.

Different self-created scenario files and CBR files are used for simulation through TCL file. Each scenario file varies in pause time for 10 to 50 and CBR also changes according to pause time. In our study favourable results are concluded because it totally depends on simulation and analysis through different method. We have generated graph for each performance metric and with help of these of these graph we have compared AODV with DSDV and AODV with DSR according to performance metrics. Every ad hoc routing protocol has its some advantages and disadvantages. Our graph shows that With respect to pause time DSR performance is overall better than AODV and DSR. This is because the network is less stressful and less dense. Ratio of sent to received packets and packet delivery fraction is also better of DSR with minimum routing load. DSDV has minimum normalized routing load because of less mobility, it use the information stored in routing table but at the same time DSDV has maximum values for the performance metrics like average end to end delay. Average throughput of all protocols is almost the same.

\section{References}

[1] C. Perkins, E. Belding-Royer, and S. Das, "Adhoc on-demand Distance vector (AODV) Routing", July 2003. Proceedings of the 2 IEEE Workshop on Mobile Computing Systems and Applications (WMCSA) (pp.90-100).

[2] D. B. Johnson, D. A. Maltz, and Y. Hu, "The dynamic source Routing protocol for mobile Adhoc networks (DSR)", April 2003.IETF Internet Draft, draft- ietf-manet-dsr-09.txt,(work in progress).

[3] Hemanth Nerra, Yufei Cheng, Egemen K, Cetenakia, Justin R. Rohar, "DSDV routing protocol implementation in ns3", SIMUTools '11 Proceedings of the 4th International ICST Conference on Simulation Tools and Techniques.

[4] Staub.T., (2004). "Ad-hoc and Hybrid Networks: Performance Comparison of MANET Routing Protocols in Ad-hoc and Hybrid Networks". Institute of Computer Science and Applied Mathematics, University of Berne, Switzerland, pp.1-38.

[5] Azizol Abdullah, Norlida Ramly, Abdullah Muhammed, Mohd Noor Derahman, "Performance Comparison Study of Routing Protocols for Mobile Grid Environment", IJCSNS International Journal of Computer Science and Network Security, Debashmita Mitra Computer Science and Engineering, CSVTU, India February 2008.

[6] S. D. Khatawkar, K. K. Pandyaji, R. M. Patil, V. M. Sali, U. L. Kulkarni, "Performance Comparison of DSDV, AODV, DSR, Routing protocols for MANET", International Conference on Computer Networks 
and Communication Systems (CNCS 2012) IPCSIT vol.35(2012) (C) (2012) IACSIT Press, Singapore, 2012.

[7] Azizol Abdullah, Norlida Ramly, Abdullah Muhammed, Mohd Noor Derahman, "Performance Comparison Study of Routing Protocols for Mobile Grid Environment", IJCSNS International Journal of Computer Science and Network Security, Debashmita Mitra Computer Science and Engineering, CSVTU, India VOL.8 No.2, pp 82-88, February 2008.

[8] Z.Alexander, "Performance Evaluation of AODV Routing Protocol", Proceedings of the third international conference on trends in technology and engineering, SCC, June 2003, pp. 133- 137.

[9] Ripan Kumar. "PERFORMANCE COMPARISON OF AODV AND DSR ROUTING PROTOCOLS IN MANETs".

[10] David B. Johnson and David A. Maltz, "Dynamic source routing in adhoc wireless networks," in Mobile computing, T. lmielinski and H. Kmh, Eds, Kluwer Academic, 1996, ch.5.

[11] C.E Perkin and P. Bhagwat. Highly Dynamic Destination-Sequenced Distance-Vector Routing (DSDV) for mobile computer. In Proceedings of the ACM Conference on Communication Architectures, Protocols and Application (SIGCOMM), pages 234-244, 1994.

[12] http://bura.brunel.ac.uk/handle/2438/4156

[13] Rahman, R.A.; Kassim, M.; Yahaya, C.K.H.C.K.; Ismail, M., "Performance analysis of routing protocol in WiMAX network," System Engineering and Technology (ICSET), 2011 IEEE International Conference on, vol., no., pp.153,157, 27-28 June 2011

[14] J. Broch, D. A. Maltz, D. B. Johnson, Y-C. Hu, and J.Jetcheva, “A performance comparison of multi-hop wireless ad hoc network routing protocols”, Mobicom' 98, October 1998, pages 85-97.

[15] S.R. Das, C.E. Perkins, and E.E. Royer, "Performance Comparison of Two On-Demand Routing Protocols for Ad Hoc Networks," Proc. INFOCOM, 2000, pp.3- 12.

[16] S. Gupta, C. Kumar, S Rani and B. Bhushan, "Performance comparison of routing protocols using different mobility models", IJMECS, vol. 4, no. 8, pp.54-61, 2012.

[17] S.S.Kaushik \& p.r.deshmukh, "Comparison of effectiveness of aodv, dsdv and dsr routing protocols in mobile ad hoc networks", International Journal of Information Technology and Knowledge Management, Vol. 2(2), pp.499-502, 2009.

[18] B. Bhushan, S. Gupta, C.K.Nagpal, “comparison of on Demand Routing Protocol”, International Journal of Information Technology and Computer Science (IJITCS), PP.61-68, DOI: 10.5815/ijitcs, 2013.

[19] Azizol Abdullah, Norlida Ramly, Abdullah Muhammed, Mohd Noor Derahman, "Performance Comparison Study of Routing Protocols for Mobile Grid Environment", IJCSNS International Journal of Computer Science and Network Security, Debashmita Mitra Computer Science and Engineering, CSVTU, India VOL.8 No.2, pp 82-88, February 2008.

[20] Z.Alexander, "Performance Evaluation of AODV Routing Protocol", Proceedings of the third international conference on trends in techologly and engineering, SCC, June 2003.

[21] Salehi, M.; Samavati, H., "DSR vs OLSR: Simulation Based Comparison of Ad Hoc Reactive and Proactive Algorithms under the Effect of New Routing Attacks," Next Generation Mobile Applications, Services and Technologies (NGMAST), 2012 6th International Conference on, vol., no., pp.100,105, 1214 Sept. 2012.

[22] Saluja, R.K.; Shrivastava, R., "A scenario based approach for gateway discovery using Manet routing protocol," Computer Communication and Informatics (ICCCI), 2012 International Conference on, vol., no., pp.1,6, 10-12 Jan. 2012

[23] H.Zafar, N.Alhamahmy, D.Harle and I.Andonovic, "Survey of Reactive and Hybrid Routing Protocols for Mobile Ad Hoc Networks", International Journal of Communication Networks and Information Security (IJCNIS), Vol. 3, No. 3, December 2011. 


\section{Author(s) Profiles}

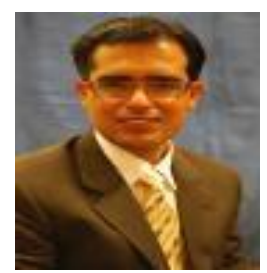

Zafar Mehmood Khattak has received his BS degree from Peshawar University, MCS degree from Kohat University in 2006, MSCS degree from University of Gujrat in 2013. Currently he is working is Lecture Compuer Science/Deputy Manger ORIC in University of Gujrat. He completed his MSCS in Wireless Networking, his research interest included Mobile Ad-hoc Networking, Wireless Sensor Network computational intelligence.

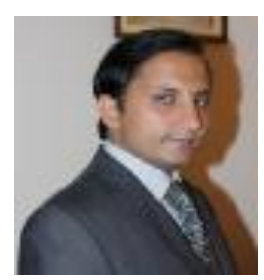

Dr. Muddesar Iqbal has done PhD from Kingston University UK in the area of Wireless Mesh Networks entitled "Design, Development and Implementation of a High Performance Wireless Mesh Network for Application in Emergency and Disaster Recovery". He has undertaken research project both in the area of healthcare and disaster recovery. He won another Award of Appreciation from ABE UK for tutoring the prize winner (from 63 countries and 541 Ins tutes/Colleges) in Informa on System Project management module in 2010. He has published 13 papers, all in Interna onal Journals and proceedings in the area of Wireless Networks targeting its application in Healthcare and Emergency and Disaster Recovery.

Muhammad Awais Nawaz has received his BS degree from University of Gujrat in Information technology, currently he is working in a software house in Dubai.

Muhammad Saleem khan is a Phd scholor at Comsat University Islamabad. He received his Master degree from Kohat University, MS degree from Muhammad Ali Jinnah University. His research interst spans over wireless technology, including Mobile Ad-hoc Networking, Wireless Sensor Network computational intelligence.

Zia UI Haq Choudhari has received his Master degree from Panjab University. Currently he is working is system administrator in University of Gujrat, Pakistan.

How to cite this paper: Zafar Mahmood, Muhammad Awais Nawaz, Mudassar Iqbal, Saleem Khan, Zia ul Haq,"Varying Pause Time Effect on AODV, DSR and DSDV Performance", IJWMT, vol.5, no.1, pp.21-33, 2015.DOI: $10.5815 /$ ijwmt.2015.01.02 